\title{
Una nueva topología de bajo costo para microinversores
}

\section{A new low-cost topology for microinverters}

\author{
SORIANO-AVENDAÑO, Luis Arturo†*, RODRÍGUEZ-CRUZ, José Luis, HIGUERA-GONZÁLEZ, \\ Rogelio M. y RIVERA-OLVERA, Jesús Noé
}

Tecnológico de Estudios Superiores de Ixtapaluca, División Ingeniería en Electrónica, Km. 7 de la carretera IxtapalucaCoatepec s/n San Juan, Distrito de Coatepec, Ixtapaluca, Estado de México C.P.56580, Tel. (055) 13148150 ext. 129

ID 1 ${ }^{\mathrm{er}}$ Autor: Luis Arturo, Soriano-Avendaño / ORC ID: 0000-0003-3299-9851, CVU CONACYT ID: 335718

ID $1^{\text {er }}$ Coautor: José Luis, Rodríguez-Cruz / ORC ID: 0000-0003-3215-2036, CVU CONACYT ID: 1016604

ID $2^{\text {do }}$ Coautor: Rogelio Manuel, Higuera-González / ORC ID: 0000-0003-3947-242X, CVU CONACYT ID: 547074

ID $3^{\text {er }}$ Coautor: Jesús Noé, Rivera-Olvera / ORC ID: 0000-0001-5425-7125, CVU CONACYT ID: 267825

DOI: $10.35429 /$ JTD.2019.12.3.7.15

Recibido: 24 de Octubre, 2018; Aceptado 11 de Diciembre, 2018

\begin{abstract}
Resumen
Recientemente, la implementación de instalaciones de energía solar ha crecido, ya que es considerada una forma amigable con el medio ambiente para producir energía eléctrica. La energía solar puede ser aprovechada bajo diferentes configuraciones o esquemas de conexión. Los esquemas de conexión con mayor aprovechamiento son la conexión en cadena, los optimizadores de potencia y los microinversores. Los microinversores poseen una amplia ventaja en comparación a los otros esquemas debido a la capacidad que tienen para aprovechar la energía solar bajo diferentes condiciones ambientales, tales como el sombreado parcial, la temperatura y la irradiación. La implementación de microinversores se ha visto frenada principalmente a causa de su alto costo y bajo tiempo de vida. De forma general, el diseño de microinversores comúnmente incluyen elementos como lo son transistores, capacitores electrolíticos, sensores y un microcontrolador. Los capacitores electrolíticos son muy utilizados como etapas de desacoplamiento, aunque presentan un tiempo de vida bajo debido a las altas frecuencias de operación, rizos de corriente. En este trabajo se presenta una nueva topología de microinversor, la cual utiliza un arreglo de capacitores multicapa en paralelo con el objetivo de reducir el costo e incrementar el tiempo de vida del microinversor.
\end{abstract}

Microinversor, energía solar, capacitores

\begin{abstract}
In recent years, the implementation of solar energy facilities has grown, as it is considered an environmentally friendly way to produce electricity. Solar energy can be exploited under different configurations or connection schemes. The most useful connection schemes are the string connection, the power optimizers and the microinverters. Microinverters have a wide advantage compared to the other schemes due to their ability to take advantage of solar energy under different environmental conditions, such as partial shading, temperature and irradiation. The implementation of microinverters has been slowed down because of its high cost and reduced lifetime. In general, the design of microinverters commonly includes elements such as transistors, electrolytic capacitors, sensors and a microcontroller. Electrolytic capacitors are commonly used as decoupling stages, even though they present a lower lifetime due to high frequencies of operation and current rises. In this paper, a new microinverter topology is presented, it uses a low-cost array of multilayer capacitors to filter high frequencies and get a low-cost topology.
\end{abstract}

Microinverters, solar energy, capacitors

\footnotetext{
Citación: SORIANO-AVENDAÑO, Luis Arturo, RODRÍGUEZ-CRUZ, José Luis, HIGUERA-GONZÁLEZ, Rogelio M. y RIVERA-OLVERA, Jesús Noé. Una nueva topología de bajo costo para microinversores. Revista del Desarrollo Tecnológico. 2019 3-12: 7-15
}

\footnotetext{
* Correspondencia del Autor (Correo electrónico: larturo.soriano@gmail.com)

$\uparrow$ Investigador contribuyendo como primer autor.
} 


\section{Introducción}

En la actualidad, la energía eléctrica es uno de los elementos esenciales para el desarrollo económico y social, ya que permite mejorar y prolongar el desempeño de las actividades del hombre más allá de las horas de luz natural. La producción de energía eléctrica de forma convencional es decir mediante el uso de recursos fósiles habilita la necesidad de buscar otras formas de aprovechamiento, ya que los efectos causados por dicha producción son uno de los principales factores de la generación del cambio climático.

La preocupación de gobiernos, industrias, y personas en general por el cambio climático y el aumento de las emisiones de dióxido de carbono al medio ambiente ha incrementado el interés por el uso y explotación de las fuentes de energía sostenibles. Las limitadas reservas de combustibles fósiles y su afectación al medio ambiente durante producción de energía eléctrica han derivado en un acelerado desarrollo e implementación de tecnologías para el aprovechamiento de energía renovable (Quinzo Lucas, Luis Fernando, 2019).

Es por esto que la posibilidad de generar energía eléctrica a partir de recursos renovables que se encuentren en una gran disponibilidad ha ganado un gran interés. Debido a esto, la producción de energía a partir de fuentes renovables ha crecido de forma exponencial a lo largo de todo el mundo (Salazar \& Garzón, 2018).

Así, la fácil instalación y formas de aprovechamiento de la energía solar son características que le han permitido presentar un mayor aprovechamiento en diferentes lugares del mundo (Torres Puentes \& Zamudio Bohórquez, Yennyfer Lizeth, 2017). Derivado de esto las aplicaciones, implementaciones e instalaciones de los sistemas fotovoltaicos se hacen cada vez más populares a lo largo de todo el mundo.

Los sistemas fotovoltaicos están integrados por paneles fotovoltaicos los cuales generan corriente continua, la cual es acondicionada mediante convertidores DC-DC o inversores DC-AC dependiendo del uso final (Rajesh \& Carolin Mabel, 2015; Hasan, Mekhilef, Seyedmahmoudian, \& Horan, 2017).
El aprovechamiento de la energía solar frecuentemente se ve afectado por las condiciones ambientales tales como: la irradiación solar, la temperatura y el sombreado parcial.

La producción de energía eléctrica a partir de energía solar, en niveles industriales y residenciales se realiza bajo diferentes esquemas de conexión de paneles fotovoltaicos (Muttaqi, Islam, \& Sutanto, 2019).

Las configuraciones de conexión de paneles fotovoltaicos más frecuentes son el arreglo tipo cadena, los optimizadores de potencia y los microinversores (Cossoli, Pedro, Firman, Andres, Cáceres, Manuel, \& Busso, Arturo, 2016; Çelik, Teke, \& Tan, 2018).

Los arreglos tipo cadenas se caracterizan por el tipo de conexión en serie y/o paralelo de los paneles fotovoltaicos, los cuales a su vez son conectados a un inversor central. Este tipo de configuración presenta una baja eficiencia ya que ocurren pérdidas durante el transporte de la energía generada al módulo del inversor, además presenta dificultades para realizar el seguimiento del máximo punto de potencia y si ocurre alguna falla en los paneles se generan perdidas por desadaptación eléctrica.

La conexión de optimizadores de potencia instala un convertidor DC-DC en cada uno de las conexiones en cadena de los paneles fotovoltaicos o en cada panel fotovoltaico, con el objetivo de minimizar los problemas de seguimiento de máximo punto de potencia, además se genera un bus de corriente continua al cual se conectan los convertidores DC-DC, el bus conduce la tensión generada a un inversor central.

La conexión basada en microinversores integra las ventajas de las configuraciones anteriores además de incrementar la eficiencia en la producción de la energía eléctrica (Sher \& Addoweesh, 2012; Ozturk \& Cadirci, 2018). En esta configuración cada panel fotovoltaico incluye su propio convertidor DC-DC e inversor $\mathrm{DC}-\mathrm{AC}$ en un diseño denominado microinversor, el cual incluye un algoritmo de seguimiento de máxima potencia o MPPT por sus siglas en inglés Maximum Power Point Tracking (Lyden \& Haque, 2015). 
La conexión de cada uno de los microinversores genera un bus de AC, lo cual deriva en una mejora en la eficiencia y se evitan las perdidas por desadaptación eléctrica. Algunas de las ventajas que presenta esta configuración es el bajo costo de instalación, el cual es menor en comparación con otras configuraciones, además de que se prescinde de circuitos de protección y de personal especializado para su instalación. Sin embargo, el mercado de los esquemas de conexión basados en microinversores se ha visto limitado debido al alto costo y bajo tiempo de vida que presenta cada uno de los módulos (Petreuş et al., 2013).

El alto costo está orientado al número de elementos que incluye el diseño del microinversor. Por otra parte, la reducción del tiempo de vida del microinversor está relacionado con el uso de capacitores electrolíticos en su topología, los cuales se utilizan para realizar aislamiento o desacoplamiento de etapas de potencia (Zhang, Xiong, Wang, Wang, \& Kong, 2017). Las topologías de los microinversores se pueden clasificar en microinversores con vinculo de corriente continua, con pseudo vinculo de corriente continua y sin vinculo de corriente continua.

Estos vínculos frecuentemente utilizan capacitores electrolíticos conectados en paralelo a las líneas de alimentación para controlar variaciones de voltaje debido a la carga y descarga, en otros casos separa los circuitos que pueden generar un mal funcionamiento o ruido en la operación del sistema (Zengin, Deveci, \& Boztepe, 2013; Lakshmi \& Hemamalini, 2018).

A este tipo de desacoplamientos también se conoce como arreglos de capacitores en derivación, los cuales frecuentemente son de aluminio y tantalio. Así, este tipo de capacitores son los que predominan en el diseño de microinversores debido a su gran capacitancia. Sin embargo, su miniaturización y diseño es difícil, además de que presentan problemas de sobrecalentamiento debido a las altas frecuencias de operación y los picos de corriente.

Actualmente, las topologías basadas en pseudo vinculo de corriente continua presentan ciertas ventajas tales como baja cantidad de elementos, simplicidad en el circuito y aislamiento galvánico.
En el presente trabajo se propone una nueva topología de microinversor de tipo pseudo vinculo de corriente continua, el cual está basado en un arreglo de capacitores cerámicos multicapa en paralelo, esto con el objetivo de minimizar el costo e incrementar el tiempo de vida del microinversor. En la primera sección se presenta de forma general a los elementos que integran un sistema fotovoltaico tales como el modelo matemático del panel fotovoltaico, el seguimiento del máximo punto de potencia, los microinversores y los capacitores multicapa. En la segunda sección se presentan a detalle los parámetros considerados para la simulación del panel fotovoltaico, el algoritmo de máximo punto de potencia y el microinversor. En la tercera sección se presentan los resultados de la simulación de la topología propuesta. Por último, se presentan las conclusiones del presente trabajo.

\section{Sistemas fotovoltaicos}

Los sistemas fotovoltaicos se pueden clasificar como aislados o conectados a la red. Para el aprovechamiento de la energía solar dichos sistemas se pueden configurar en diferentes esquemas de conexión, tales como conexiones en cadena, optimizadores de potencia $y$ microinversores. Siendo los esquemas de conexión basados en microinversores los que presentan un mayor, este tipo de sistemas en general están integrados por paneles fotovoltaicos, un algoritmo de MPPT y un microinversor como se muestra en la figura 1 .

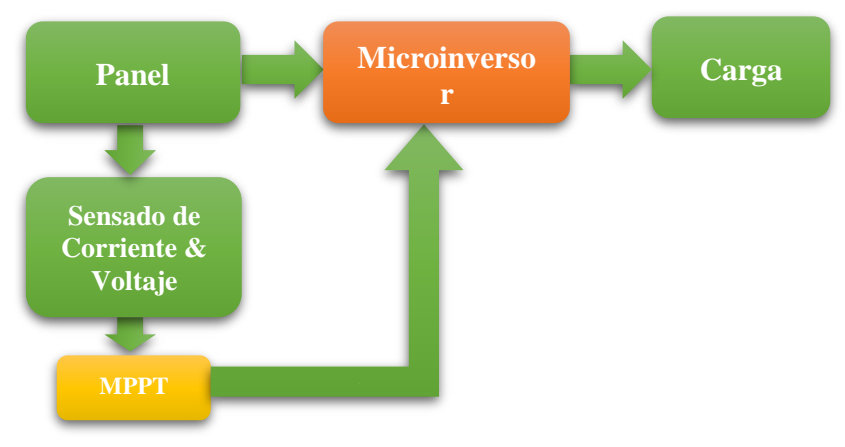

Figura 1 Sistema fotovoltaico basado en microinversor Fuente: Elaboración Propia

\section{A. Modelo matemático del panel solar}

La transformación de energía solar en energía eléctrica se realiza mediante paneles solares, el cual ésta integrado por celdas fotovoltaicas conectadas en serie y paralelo que absorben los fotones mediante las uniones de material p-n y liberan electrones.

SORIANO-AVENDAÑO, Luis Arturo, RODRÍGUEZ-CRUZ, José Luis, HIGUERA-GONZÁLEZ, Rogelio M. y RIVERA-OLVERA, Jesús Noé. Una nueva topología de bajo costo para microinversores. Revista del Desarrollo Tecnológico. 2019 
Este comportamiento se puede describir a través de modelos matemáticos, actualmente existen un gran número de modelos para paneles fotovoltaicos (Koohi-Kamali, Rahim, Mokhlis, \& Tyagi, 2016). El presente trabajo está basado en el modelo de un diodo presentado en (Bellia, Youcef, \& Fatima, 2014), el cual es una muy buena aproximación, este modelo es presentado en la figura 2.

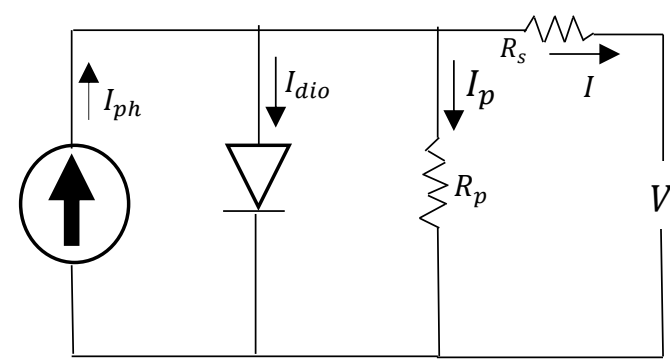

Figura 2 Modelo de una celda fotovoltaica Fuente: Elaboración Propia

A partir de la ley de corrientes de Kirchoff la relación del circuito mostrado en la figura 2 resulta como se muestra en la siguiente ecuación

$I=I_{p h}-I_{d i o}-I_{P}$

En donde $I_{p h}$ es la fotocorriente generada por la celda cuando es expuesta a los rayos del sol, $I_{\text {dio }}$ es la corriente del diodo en paralelo y $I_{P}$ es la corriente del resistor $R_{P}$ en derivación. Substituyendo $I_{d i o}$ y $I_{P}$ en (1) se obtiene

$$
I=I_{p h}-I_{0}\left[\exp \left(\frac{V+I R_{S}}{A V_{T}}\right)-1\right]-\frac{V+I R_{S}}{R_{p}}
$$

En donde $V_{T}=\frac{k T}{q}, q=1.602 \times 10^{-19} \mathrm{C}$ es la carga electrónica, $k=1.38 \times 10^{-23} \mathrm{~J} / \mathrm{K}$ es la constante de Boltzmann, $A$ es el factor de idealidad del diodo y $T$ es la temperatura de la celda. El modelo matemático de una celda fotovoltaica se puede extender al modelo de un panel fotovoltaico, el cual comúnmente considera conexiones de cierto número de celdas en serie $N_{s}$ y paralelo $N_{p}$. Por lo que el comportamiento de un panel fotovoltaico se puede obtener mediante el modelo que se describe en (3) como.

$I=I_{p h}-I_{0}\left[\exp \left(\frac{V+I R_{S} \frac{N_{S}}{N_{p}}}{A V_{T} N_{S}}\right)-1\right]-\frac{V+I R_{S} N_{S_{p}}}{\frac{N_{S}}{N_{p}} R_{p}}$

\section{B. Seguimiento del máximo punto de potencia}

La producción de energía eléctrica mediante paneles fotovoltaicos frecuentemente se ve afectada por las condiciones ambientales tales como la temperatura, la irradiación solar y el sombreado parcial. Es por esto que los sistemas para el aprovechamiento de la energía solar incluyen un algoritmo de máximo punto de potencia. Este algoritmo tiene por objetivo buscar el punto en donde ocurre la máxima potencia. Se han desarrollado un gran número de algoritmos de seguimiento de máximo punto de potencia, estos se pueden clasificar en técnicas convencionales y no convencionales. Las técnicas heurísticas, metaheurísticas y de inteligencia artificial son consideradas técnicas no convencionales (Eltawil \& Zhao, 2013; Karami, Moubayed, \& Outbib, 2017).

El seguimiento del máximo punto de potencia está basado en la curva del gráfico 1, la cual muestra la gráfica de voltaje contra potencia, la cual depende de las condiciones ambientales antes mencionadas.

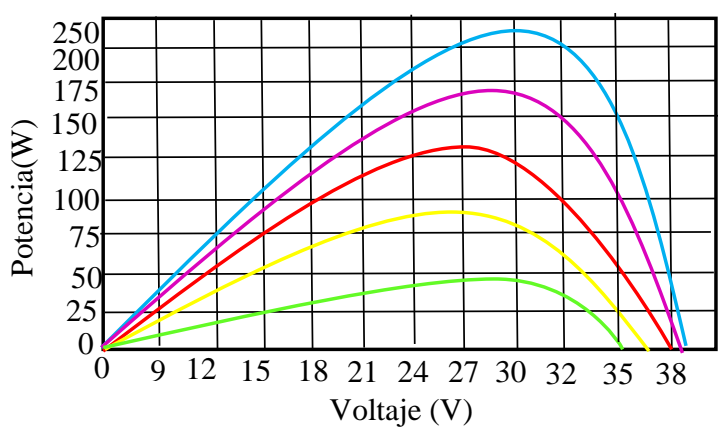

Gráfico 1 Curva voltaje-potencia

Fuente: Elaboración Propia

Esto quiere decir que la curva a lo largo del tiempo cambia de forma por lo que es difícil saber cuáles son las condiciones en las cuales se puede extraer la mayor cantidad de energía. En el gráfico 1 se muestra diferentes cambios de irradiación, estos cambios afectan el voltaje de referencia el cual le indica al microinversor a través del ciclo de trabajo la potencia a extraer.

\section{Microinversores}

Un microinversor es un dispositivo que transforma la energía de corriente continua generada por los paneles fotovoltaicos a corriente alterna. El microinversor esta basado en las topologías de los convertidores DC-DC y en los inversores DC-AC. 
Esto se traduce en una etapa de elevación de voltaje de corriente continua y una etapa de inversión de voltaje de corriente continua a alterna. Estas etapas en el microinversor son separadas o desacopladas mediante capacitores electrolíticos o aislamientos galvánicos, esto con el fin de minimizar las perdidas, daños o ruido en cada una de las etapas.

Para la inversión de voltaje frecuentemente se utilizan técnicas de conmutación convenciones y avanzadas. Los microinversores son dispositivos que son utilizados para el aprovechamiento de los sistemas fotovoltaicos conectados a la red, ya que presentan una mayor eficiencia con respecto a las conexiones de tipo cadena y optimizadores de potencia. Un microinversor se conecta a cada panel fotovoltaico y su rango de diseño está orientado para potencias de hasta $300 \mathrm{~W}$.

Las topologías actuales de microinversores se pueden clasificar de acuerdo a la disposición del vínculo de corriente continua o DC-link. En la figura 3 se muestra la clasificación basada en DC-link, en donde (a) es la topología basada en vinculo de corriente continua, (b) está basada en pseudo vinculo de corriente continua y (c) no considera el vínculo de corriente continua.

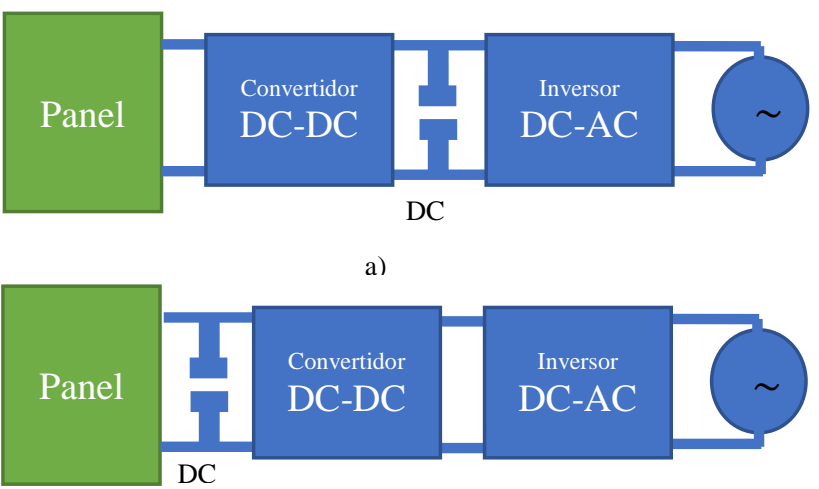

b)

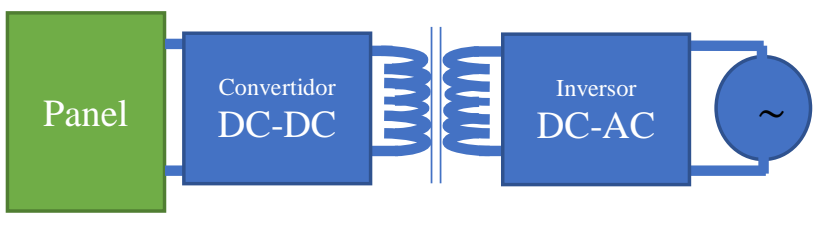

c)

Figura 3 Topologías de microinversore Fuente: Elaboración Propia

Las topologías basadas en vínculos de corriente continua requieren de técnicas de conmutación especiales para minimizar las pérdidas a altas frecuencias, las cuales aumentan la cantidad de elementos, el tamaño y costo.
Por otra parte, las topologías sin vínculos de corriente continua requieren de un gran número de elementos en comparación a las otras, utilizan técnicas de conmutación más sofisticadas que en ocasiones son difíciles de implementar. Este tipo de topologías no requieren de capacitores de grandes valores. Finalmente, las topologías basadas en pseudo vínculos de corriente continua son las que utilizan el menor número de componentes y prescinden de capacitores gran tamaño, pero presentan problemas con la generación de armónicos, lo que se puede solucionar con técnicas de conmutación avanzada.

\section{Capacitores cerámicos multicapa}

La miniaturización y los grandes avances tecnológicos han permitido diseñar capacitores cerámicos multicapa (MLCC por sus siglas en inglés Multilayer Ceramic Chip Capacitor) de gran capacitancia como se puede ver en la figura 4. Estos se pueden clasificar en dos categorías de acuerdo al tipo de material cerámico dieléctrico utilizado, la clase 1 y 2 están diseñados a bajas y altas capacitancias respectivamente. La clase 1 también se conocen como compensadores de temperatura y la clase 2 como capacitores de constante dieléctrica alta.

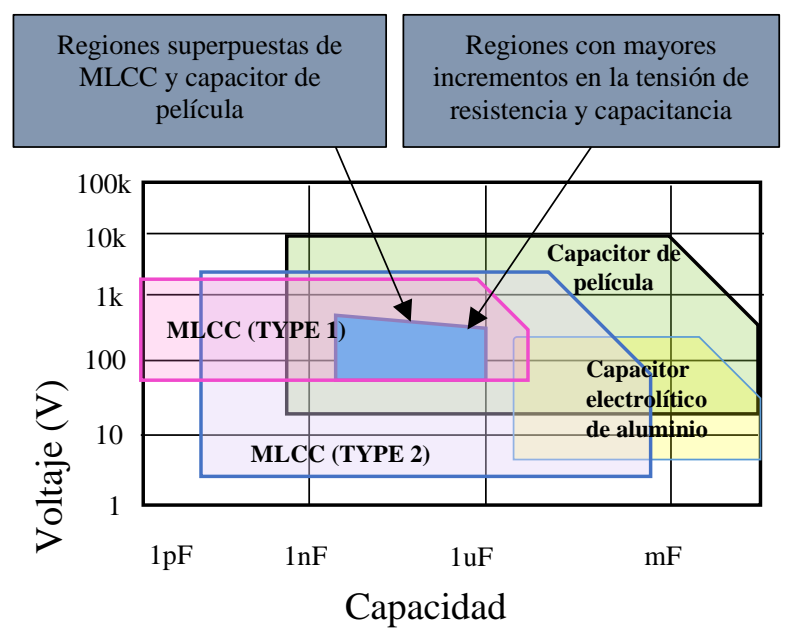

Figura 4 Regiones de voltaje-capacitancia de diferentes capacitores (TDK Corporation, 2019)

De acuerdo a (Murata Manufacturing Co., Ltd., n.d.) el desarrollo de capacitores multicapa se incrementara en valores de capacitancia así como el rango de voltajes en donde se puedan aplicar. Esto se debe principalmente a las ventajas que presenta con respecto a los otros tipos de capacitores. En la tabla 1 se presentan las ventajas y desventajas de los capacitores de película, electrolíticos de aluminio y multicapa de clase 2 . 


\begin{tabular}{|c|c|c|c|}
\hline Características & $\begin{array}{l}\text { Cerámica } \\
\text { multicapa }\end{array}$ & Película & Electrolítico \\
\hline Capacitancia & $x$ & () & () \\
\hline Frecuencia & ○) & $\Delta$ & $x$ \\
\hline Temperatura & () & ○) & $x$ \\
\hline Voltaje & $\Delta$ & () & $\Delta$ \\
\hline $\begin{array}{l}\text { Generación de } \\
\text { calor }\end{array}$ & ○) & () & $x$ \\
\hline Tamaño & () & $x$ & $\Delta$ \\
\hline $\begin{array}{ll}\text { Tiempo } & \text { de } \\
\text { vida } & \\
\end{array}$ & ○ & () & $\times$ \\
\hline Costo & ○ & $x$ & () \\
\hline
\end{tabular}

Tabla 1 Comparación y caracteristicas de los principales capacitores (Murata Manufacturing Co., Ltd., n.d.; TDK Corporation, 2019)

\section{Metodología}

El diseño propuesto incluye la simulación de un panel fotovoltaico, la implementación de un algoritmo MPPT convencional y el diseño de un microinversor en Matlab ${ }^{\mathrm{TM}}$.

\section{A. Simulación del panel fotovoltaico}

Las características del panel fotovoltaico considerado en este trabajo es el Diamond Premium MLE 275W de Mitsubishi Electric US, Inc. La tabla 2 muestra sus características.

\begin{tabular}{|l|l|}
\hline \multicolumn{1}{|c|}{ Nombre del modelo } & \multicolumn{1}{c|}{$\begin{array}{c}\text { PV- } \\
\text { MLE275HD2 }\end{array}$} \\
\hline Tipo de celda & Monocrystalline \\
\hline Número de celdas & 120 Cell \\
\hline Potencia máxima nominal (Pmax) & $275 \mathrm{~W}$ \\
\hline Corriente de cortocircuito (Isc) & $9.28 \mathrm{~A}$ \\
\hline $\begin{array}{l}\text { Tensión de potencia máxima } \\
\text { (Vmp) }\end{array}$ & $32.1 \mathrm{~V}$ \\
\hline $\begin{array}{l}\text { Corriente máxima de potencia } \\
\text { (Imp) }\end{array}$ & $8.58 \mathrm{~A}$ \\
\hline $\begin{array}{l}\text { Temperatura normal de } \\
\text { funcionamiento de la celda } \\
\text { (NOCT) }\end{array}$ & $46.5^{\circ} \mathrm{C}$ \\
\hline $\begin{array}{l}\text { Dimensiones } \\
(1625 \times 1019 \mathrm{x} \\
46 \mathrm{~mm})\end{array}$ \\
\hline
\end{tabular}

Tabla 2 Especificaciones del panel PV-MLE275HD2 Fuente: Elaboración Propia

\section{B. Algoritmo MPPT}

El algoritmo de seguimiento de máximo punto de potencia tiene por objetivo asegurar que ante los cambios en las condiciones medio ambientales como lo es la temperatura e irradiación la máxima potencia disponible en el panel sea extraída.
El algoritmo frecuentemente comanda el ciclo de trabajo de la etapa de conversión de corriente continua del microinversor para encontrar el punto de operación que converja con el punto de máxima potencia de la curva voltajepotencia.

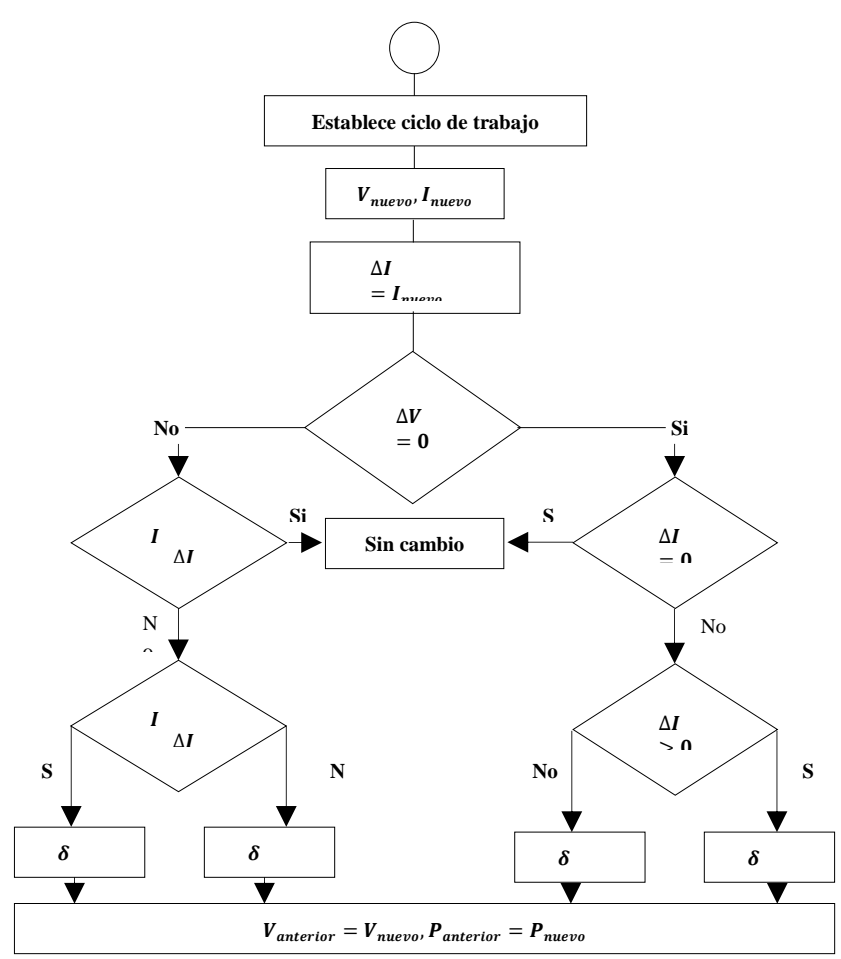

Figura 5 Algoritmo de conducta incremental para MPPT Fuente: Elaboración Propia

Los algoritmos MPPT se caracterizan por obtener el máximo punto de operación, una rápida velocidad de seguimiento, no oscilar alrededor del máximo punto de potencia y su fácil implementación, esto con el objetivo de incrementar la eficiencia del sistema.

El algoritmo de conducta incremental (IC por sus siglas en inglés Icremental Conductance) es uno de los algoritmos convencionales, que busca el máximo punto de potencia a través de la derivada de la potencia con respecto del voltaje, en donde el resultado es 0 .

De acuerdo a la figura 5 el máximo punto de potencia puede ser alcanzado comparando la relación entre el voltaje y la corriente de la conducta instantánea contra su derivada o conducta incremental. Si se encuentra que la derivada es nula entonces el ciclo de trabajo se encuentra en el máximo punto de potencia.

Por otra parte, si es mayor a cero se encuentra a la derecha del MPP y si es menor a cero a la izquierda del MPP. 


\section{Diseño del microinversor}

El diseño de microinversor propuesto es del tipo pseudo vinculo de corriente continua y está basado en (Kasa, Iida, \& Chen, 2005), debido al bajo número de elementos que se involucran. El diseño propuesto agrega un arreglo de capacitores multicapa en paralelo a la entrada tal como se muestra en la figura 6 .

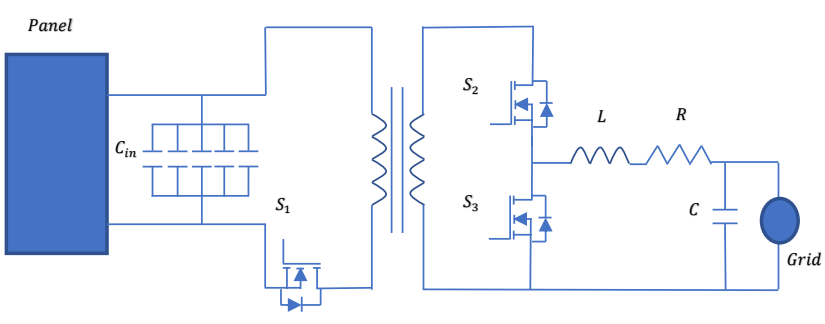

Figura 6 Diseño de microinversor propuesta Fuente: Elaboración Propia

Como se mencionó anteriormente la diferencia primordial del diseño propuesto de pseudo vinculo de corriente continua está formado por un arreglo de capacitores en paralelo de tipo MLCC, con el objetivo de reducir el costo e incrementar el tiempo de vida del microinversor.

Debido a la limitación en los valores de capacitancia del MLCC los cuales van desde el orden de los $p F$ hasta los $100 \mu F$ de forma comercial se propone un arreglo en paralelo para obtener una capacitancia del orden de los 1000 $\mu F$ en el pseudo vinculo mostrado en la figura 6 .

La etapa de conversión de corriente continua está basada en un convertidor del tipo flyback, el cual tiene una configuración similar a la del convertidor elevador-reductor.

El convertidor flyback cuenta con un aislamiento galvánico que acopla dos bobinas con valores de $300 \mathrm{mH}$. Las terminales del panel son conectadas al primario y a un interruptor de tipo transistor de efecto de campo metal-óxido o MOSFET (por sus siglas en inglés Metal-oxidesemiconductor field-effect transistor).

El interruptor es modulado por ancho de pulso en donde el ciclo de trabajo es definido por el algoritmo de MPPT. Para la transformación de la energía de corriente continua se utiliza un inversor de medio puente, el cual está integrado por dos MOSFET y es conmutado por la técnica de modulación senoidal de ancho de pulso.
Por lo que el diseño del inversor de medio puente considera los siguientes parámetros una frecuencia deseada de $60 \mathrm{~Hz}$, una frecuencia de conmutación de $10 \mathrm{KHz}$, un filtro de segundo orden pasa bajas con $R=1 \Omega$, $L=300 \mathrm{mH}$ y $C=50 \mu \mathrm{f}$.

\section{Resultados}

El modelo matemático del panel fue diseñado en Matlab Simulink y la topología del inversor en Matlab Simscape. Los parámetros definidos para la simulación se muestran en la tabla 3

\begin{tabular}{|l|l|}
\hline \multicolumn{1}{|c|}{ Parámetro } & \multicolumn{2}{c|}{ Valor } \\
\hline Tipo de simulación & Discreta \\
\hline Tiempo de muestreo & $1 \mathrm{e}-6$ \\
\hline Solucionador & Ode $15 \mathrm{~s}(\mathrm{Stiff} / \mathrm{NDF})$ \\
\hline
\end{tabular}

Tabla 3 Parámetros de simulación

Fuente: Elaboración Propia

Para la simulación de las condiciones del medio ambiente, es decir la irradiación y la temperatura se consideró el perfil mostrado en el gráfico 2.

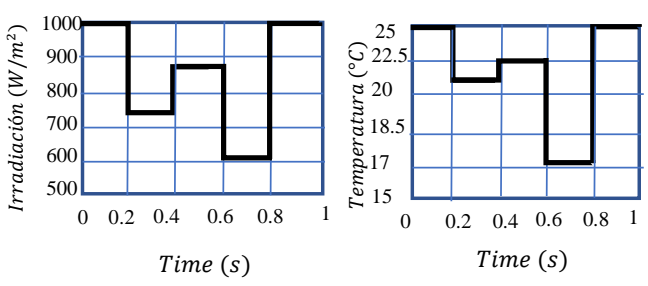

Gráfico 2 Perfil de irradiación y temperatura Fuente: Elaboración Propia

La conmutación del interruptor $S_{1}$ está definida como $20 \mu \mathrm{s}$. La conmutación de $S_{2}$ y $S_{3}$ está definida en $100 \mu s$.

La salida del inversor de medio puente basada en la modulación senoidal de ancho de pulso se muestra en el grafico 3. Como se puede ver en el grafico 3, con el diseño propuesto se obtuvieron la salida en voltaje y corriente del microinversor es a $60 \mathrm{~Hz}$.

El diseño propuesto es de bajo costo ya que la tecnología de capacitores multicapa presenta precios similares o más bajos a la tecnología de capacitores electrolíticos tal como se muestra en la tabla 4. Por último, al no utilizar capacitores electrolíticos los cuales son unas de las principales causas del bajo tiempo de vida de los microinversores, el microinversor propuesto presenta un incremento en el tiempo de vida. 

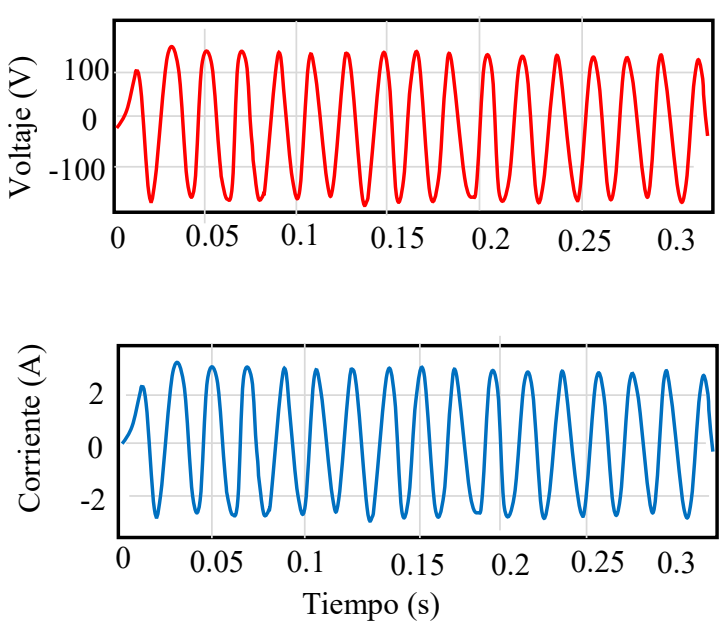

Gráfico 3 Voltaje y corriente de salida del microinversor propuesto

Fuente: Elaboración Propia

\begin{tabular}{|l|l|l|l|}
\hline \multicolumn{1}{|c}{$\begin{array}{l}\text { Tipo de } \\
\text { capacitor }\end{array}$} & \multicolumn{1}{c|}{ Modelo y Marca } & Capacidad \\
\hline $\begin{array}{l}\text { Capacitor de } \\
\text { película }\end{array}$ & $\begin{array}{l}\text { B32524R0107K000 } \\
\text { (EPCOS-TYDK) }\end{array}$ & $\begin{array}{l}\text { \$13.25 } \\
\text { USD }\end{array}$ & $100 \mathrm{uF}-63 \mathrm{~V}$ \\
\hline Electrolítico & $\begin{array}{l}\text { 661EKY500D101M } \\
\text { HB5D } \\
\text { (UNITED CHEMI- } \\
\text { CON) }\end{array}$ & $\begin{array}{l}\text { \$0.702 } \\
\text { USD }\end{array}$ & 100uF-50V \\
\hline $\begin{array}{l}\text { Capacitor } \\
\text { cerámico } \\
\text { multicapa }\end{array}$ & $\begin{array}{l}\text { ST205C107MAL10 } \\
\text { (AVX) }\end{array}$ & $\begin{array}{l}\text { \$55.47 } \\
\text { USD }\end{array}$ & $100 \mathrm{uF}-50 \mathrm{~V}$ \\
\hline $\begin{array}{l}\text { Capacitor } \\
\text { cerámico } \\
\text { multicapa }\end{array}$ & $\begin{array}{l}\text { CL31A106MBHNN } \\
\text { NE } \\
\text { (Samsung) }\end{array}$ & $\begin{array}{l}\text { \$0.30 } \\
\text { USD }\end{array}$ & $10 \mu \mathrm{F}-50 \mathrm{~V}$ \\
\hline
\end{tabular}

Tabla 4 Costos de capacitores

Fuente: Elaboración Propia

\section{Trabajo futuro}

El aprovechamiento de la energía solar mediante esquemas de conexión basados en microinversores es muy prometedora, debido principalmente a sus ventajas contra otros esquemas de conexión en sistemas fotovoltaicos. Como trabajo futuro se espera desarrollar nuevas topologías con un menor número de elementos de electrónica de potencia y además diseñar nuevas técnicas de control que permitan minimizar las perdidas por condiciones ambientales al mismo tiempo que se diseñan a bajas frecuencias de conmutación, ya que el uso de altas frecuencias altas en el control de microinversores pueden ser perjudiciales en el tiempo de vida de los semiconductores.

El diseño de controladores con un menor número de elementos son uno de los principales objetivos en el diseño de microinversores.

\section{Conclusiones}

El presente trabajo presenta una nueva topología de microinversor, la cual se simulo y analizo un panel fotovoltaico comercial, un algoritmo de seguimiento de máxima potencia y un microinversor. La nueva topología está basada en un puente de capacitores multicapa en paralelo. Esto con el objetivo de incrementar el tiempo de vida de los microinversores, la cual frecuentemente se ve reducida por el bajo tiempo de vida de los capacitores electrolíticos. Los resultados muestran que se obtienen una salida de la misma calidad utilizando un arreglo de capacitores multicapa que un capacitor electrolítico, así esto a su vez deriva en un incremento en el tiempo de vida de los micro inversores.

\section{Referencias}

Bellia, H., Youcef, R., \& Fatima, M. (2014). A detailed modeling of photovoltaic module using MATLAB. NRIAG Journal of Astronomy and Geophysics, 3(1), 53-61. https://doi.org/10.1016/j.nrjag.2014.04.001

Çelik, Ö., Teke, A., \& Tan, A. (2018). Overview of micro-inverters as a challenging technology in photovoltaic applications. Renewable and Sustainable Energy Reviews, 82, 3191-3206. https://doi.org/10.1016/j.rser.2017.10.024

Cossoli, Pedro, Firman, Andres, Cáceres, Manuel, \& Busso, Arturo. (2016). Microinversores: Principales topologías. Extensionismo, Innovación y Transferencia Tecnológica, 2, 14-21.

Eltawil, M. A., \& Zhao, Z. (2013). MPPT techniques for photovoltaic applications. Renewable and Sustainable Energy Reviews, 25, 793-813.

https://doi.org/10.1016/j.rser.2013.05.022

Hasan, R., Mekhilef, S., Seyedmahmoudian, M., \& Horan, B. (2017). Grid-connected isolated PV microinverters: A review. Renewable and Sustainable Energy Reviews, 67, 1065-1080. https://doi.org/10.1016/j.rser.2016.09.082

Karami, N., Moubayed, N., \& Outbib, R. (2017). General review and classification of different MPPT Techniques. Renewable and Sustainable Energy Reviews, 68, 1-18. https://doi.org/10.1016/j.rser.2016.09.132 
Kasa, N., Iida, T., \& Chen, L. (2005). Flyback Inverter Controlled by Sensorless Current MPPT for Photovoltaic Power System. IEEE Transactions on Industrial Electronics, 52(4), 1145-1152.

https://doi.org/10.1109/TIE.2005.851602

Koohi-Kamali, S., Rahim, N. A., Mokhlis, H., \& Tyagi, V. V. (2016). Photovoltaic electricity generator dynamic modeling methods for smart grid applications: A review. Renewable and Sustainable Energy Reviews, 57, 131-172. https://doi.org/10.1016/j.rser.2015.12.137

Lakshmi, M., \& Hemamalini, S. (2018). Decoupled control of grid connected photovoltaic system using fractional order controller. Ain Shams Engineering Journal, 9(4), 927-937.

https://doi.org/10.1016/j.asej.2016.06.002

Lyden, S., \& Haque, M. E. (2015). Maximum Power Point Tracking techniques for photovoltaic systems: A comprehensive review and comparative analysis. Renewable and Sustainable Energy Reviews, 52, 1504-1518. https://doi.org/10.1016/j.rser.2015.07.172

Murata Manufacturing Co., Ltd. (n.d.). Technical Report: Evolving CapacitorsMultilayer Ceramic Capacitors Part 2: Technology (part 1 of 2). Retrieved July 11, 2019, from https://www.murata.com/eneu/products/emiconfun/capacitor/2014/06/24/en -20140624-p1

Muttaqi, K. M., Islam, M. R., \& Sutanto, D. (2019). Future Power Distribution Grids: Integration of Renewable Energy, Energy Storage, Electric Vehicles, Superconductor, and Magnetic Bus. IEEE Transactions on Applied Superconductivity, 29(2), 1-5. https://doi.org/10.1109/TASC.2019.2895528

Ozturk, S., \& Cadirci, I. (2018). A Generalized and Flexible Control Scheme for Photovoltaic Grid-Tie Microinverters. IEEE Transactions on Industry Applications, 54(1), 505-516. https://doi.org/10.1109/TIA.2017.2753175

Petreuş, D., Daraban, S., Ciocan, I., Patarau, T., Morel, C., \& Machmoum, M. (2013). Low cost single stage micro-inverter with MPPT for grid connected applications. Solar Energy, 92, 241255.

https://doi.org/10.1016/j.solener.2013.03.016
Quinzo Lucas, Luis Fernando. (2019). Proyecto para el diseño de un sistema hibrido eólicofotovoltaico para el complejo deportivo "Cancha Sho" en la Ciudadela Bellavista de la ciudad de Guayaquil. Universidad Cátolica de Santiago de Guayaquil, Guayaquil - Ecuador.

Rajesh, R., \& Carolin Mabel, M. (2015). A comprehensive review of photovoltaic systems. Renewable and Sustainable Energy Reviews, 51, 231-248.

https://doi.org/10.1016/j.rser.2015.06.006

Salazar, W. A. B., \& Garzón, F. A. C. (2018). Propuesta de un sistema fotovoltaico para consumo eléctrico en el municipio de Quebradanegra, Cundinamarca. 92.

Sher, H. A., \& Addoweesh, K. E. (2012). Microinverters-Promising solutions in solar photovoltaics. Energy for Sustainable Development, 16(4), 389-400. https://doi.org/10.1016/j.esd.2012.10.002

TDK Corporation. (2019). MLCC (Multilayer Ceramic Chip Capacitor) C0G Guide for Resonance Circuit Vol.1. Retrieved July 11, 2019, from https://product.tdk.com/info/en/products/capacit or/ceramic/mlcc/technote/solution/mlcc04/inde x.html

Torres Puentes, C. C. T., \& Zamudio Bohórquez, Yennyfer Lizeth. (2017). Análisis de conveniencia para la implementación de la energía solar fotovoltaica en el ITD Juan del Corral en la Ciudad de Bogotá D.C. 98.

Zengin, S., Deveci, F., \& Boztepe, M. (2013). Decoupling Capacitor Selection in DCM Flyback PV Microinverters Considering Harmonic Distortion. IEEE Transactions on Power Electronics, 28(2), 816-825. https://doi.org/10.1109/TPEL.2012.2203150

Zhang, Y., Xiong, J., Wang, Z., Wang, S., \& Kong, L. (2017). Review of power decoupling methods for microinverters without using electrolytic capacitors. IECON 2017 - 43rd Annual Conference of the IEEE Industrial Electronics Society, 1493-1498. https://doi.org/10.1109/IECON.2017.8216254 